\title{
EDITORIAL
}

\section{Impact of SARS on development of childhood acute lymphoblastic leukaemia}

\author{
Leukemia (2007) 21, 1353-1356; doi:10.1038/sj.leu.2404729
}

Acute lymphoblastic leukaemia (ALL) is the commonest malignancy in children. The aetiology of childhood ALL is not clear, but it is postulated to be multiple-hit with interaction of genetic and environmental factors. One subtype of ALL, 'Common' ALL, is more common in resource-rich countries. There were studies suggesting population mixing at young age associated with higher incidence of Common ALL. ${ }^{1,2}$ There were also reports on exposure of some common infection during infancy protecting children from development of ALL in later age. ${ }^{3}$ Two local studies in Hong Kong supported the hypothesis of population mixing and the protective effect of early exposure to infection. ${ }^{4,5}$ Most of the epidemiological studies were studied in a relatively stable community without significant changes in lifestyle. In the first half of 2003, Hong Kong experienced the worst hit of severe acute respiratory syndrome (SARS) in the world. From 28 February 2003 to 2 June 2003, a total of 1755 patients were diagnosed with SARS with 300 mortalities. ${ }^{6}$ With the high number of patients infected in the community, the Hong Kong Government had instituted extraordinary measures to prevent the spread of the infection. During that period, classes were suspended and schools were closed for 2 months. After resuming classes, there were strict measures to prevent spread of communicable infections. Schools were required to cleanse and disinfect the facilities daily, and maintain good indoor ventilation. Students were required to wear masks in schools. Body temperature checking was compulsory for all students before going to school. Children with fever or respiratory infections were not allowed to attend schools. The hand-washing campaign was a major public event during the SARS period. Most people avoided going to public areas and group activities were all cancelled. The strict infection control measures lasted for about 6 months.

With the hypothesis of population mixing and exposure of common infection, the above major social changes in Hong Kong during the SARS period may have had an impact on the incidence of childhood ALL. A study was then performed to investigate any change in the incidence of childhood ALL in Hong Kong over the past 10 years with special reference to the SARS period and to compare with incidence of other types of childhood cancers during the same period. The Hong Kong Paediatric Haematology \& Oncology Study Group was formed by the paediatric oncology units in public hospitals. It started to collect childhood cancer statistics prospectively since 1994, and the data were matched with the Hong Kong Cancer Registry, which is a government-supported body collecting cancer data for the whole of Hong Kong. Since the childhood leukaemia and childhood cancers were all treated in the paediatric oncology units of public hospitals, the childhood cancer statistics to be analyzed in this study would be considered exhaustive within Hong Kong. ${ }^{7}$ The study subjects were $1-<15$ years old. Children below the age of 1 year were excluded, as infant ALL has distinct cytogenetic subtype, which is less affected by environmental factors. The trend of childhood ALL was analysed from 1994 to 2005, that is for the 10 years before SARS to 2 years after SARS. The trend was studied for all types of childhood ALL and then separately for the standard-risk ALL. Standard-risk ALL was defined according to the National Cancer Institute Workshop criteria: age 1.00-9.99 years and presenting white cell counts $<50 \times 10^{9} /$. $^{8}$ Common ALL was defined as patients with immunophenotyping of B-lineage with CD10 positive and cytoplasmic immunoglobulin negative. Children with other types of malignancy were included as control group. Childhood cancer below 1 year was also excluded, as the chance of congenital malignancy is much higher in this group. Brain tumour patients were also excluded, as clinical presentation may be more insidious, and there might be a delay in diagnosis during the SARS period.

Patients were classified into three groups, patients with standard risk of common ALL $(S R)$, patients with all other types of childhood ALL $(A L L)$ and non-ALL cancers patients (non- $A L L$ ). The non-ALL group is considered as the control group. The number of specific cancer cases by month was captured for each group. We applied the wavelet-based smoothing technique to model the cancer incidence, and visualized the trends through wavelet-based plots for each of the three groups. After that we applied the robust minimum m-estimation (MM) regression that incorporated the defined indicator variable to test if there was a significant change in the corresponding SARS period. The details of the method to test the significance of the impact of SARS on cancer incidence are presented in Supplementary Information. The number of newly diagnosed ALL cases from year 1993 to 2005 are shown in Table 1. Year 2003 had a lower number of $\mathrm{ALL}$, in particular the standard-risk ALL subtype and Common ALL below the age of 10 years. The decrease in ALL cases in 2003 was followed by an increase of new cases back to the usual range in the following 2 years. The number of non-ALL cases was quite constant over the years. By using the waveletbased smooth method, zooming in and out using the multiresolution approximations S1, S2, S3 and S4, we focused on different features of the signals. The coarse scale approximation, say S4, gives a 'faraway view' of the signal; we see the overall shape by significant changes. The fine-scale approximations, say $\mathrm{S} 1$, give a 'close in view' of the signal, revealing more details but also more noise. Clearly, S4 in Figure 1a shows two significant periodic changes (not spike change) around the years 1997 and 2003, respectively. To focus on S4 and visualize the 12 most significant features from the most significant wavelet S4.7 to S4.2, all three of the extreme (minimum) values of standard risk occurred during the SARS period in the smoothed approximation signal (Figure 2). Similar plots for ALL and non-ALL were also plotted, and no significant trends appeared at the SARS period, that is both smoothed approximation signals were quite flat and the minimum points were not in the SARS period. We then applied the robust MM-estimation with the dummy variable method. The impact of SARS period for the standardrisk and common ALL (SR) subtypes was significant as shown by the F-test for the indicator variable $D(P=0.0110)$. The impact of SARS period for ALL subtypes was of borderline significance, as shown by the F-test $(P=0.0374)$. The detailed results of the statistical analysis are shown in Supplementary Information. The number of notifiable communicable diseases reported to the 
Table 1 Number of childhood cancers in year 1993-2005 and notifiable diseases in year 1997-2005

\begin{tabular}{|c|c|c|c|c|c|c|c|c|c|c|c|c|c|c|}
\hline & 1993 & 1994 & 1995 & 1996 & 1997 & 1998 & 1999 & 2000 & 2001 & 2002 & 2003 & 2004 & 2005 & Total no. \\
\hline \multicolumn{15}{|l|}{$A L L$} \\
\hline \multirow{2}{*}{\multicolumn{15}{|c|}{ Whole group }} \\
\hline \multicolumn{10}{|l|}{ Age } & & & & & \\
\hline $1-14$ years & 28 & 30 & 31 & 37 & 29 & 28 & 29 & 32 & 41 & 31 & 25 & 35 & 29 & 405 \\
\hline $1-9.9$ years & 23 & 23 & 26 & 30 & 22 & 22 & 24 & 26 & 33 & 22 & 16 & 28 & 25 & 320 \\
\hline \multicolumn{15}{|l|}{ Common } \\
\hline \multicolumn{15}{|l|}{ Age } \\
\hline $1-14$ years & 22 & 19 & 18 & 25 & 16 & 19 & 16 & 19 & 27 & 11 & 10 & 23 & 16 & 241 \\
\hline $1-9.9$ years & 17 & 16 & 16 & 20 & 12 & 16 & 15 & 16 & 23 & 11 & 7 & 17 & 15 & 201 \\
\hline Standard risk & 21 & 19 & 20 & 28 & 18 & 18 & 24 & 24 & 30 & 21 & 16 & 25 & 23 & 287 \\
\hline \multicolumn{15}{|l|}{$T$ cell } \\
\hline \multicolumn{15}{|l|}{ Age } \\
\hline $1-14$ years & 1 & 3 & 3 & 1 & 5 & 6 & 2 & 4 & 4 & 5 & 1 & 2 & 2 & 39 \\
\hline $1-9.9$ years & 1 & 2 & 2 & 1 & 4 & 4 & 0 & 2 & 2 & 1 & 0 & 2 & 1 & 22 \\
\hline \multicolumn{15}{|c|}{ Other childhood cancer ${ }^{a}$} \\
\hline \multicolumn{15}{|c|}{ Age } \\
\hline $1-14$ years & - & 44 & 42 & 58 & 59 & 59 & 56 & 65 & 75 & 71 & 51 & 46 & 42 & 668 \\
\hline $1-9.9$ years & - & 28 & 30 & 30 & 34 & 40 & 38 & 44 & 40 & 54 & 32 & 29 & 23 & 422 \\
\hline Chickenpox & - & - & - & - & - & - & 10890 & 8556 & 16472 & 16727 & 6780 & 11784 & 11960 & \\
\hline Measles & - & - & - & - & 316 & 56 & 55 & 61 & 179 & 61 & 33 & 51 & 65 & \\
\hline Scarlet fever & - & - & - & - & 77 & 27 & 126 & 123 & 147 & 119 & 63 & 125 & 177 & \\
\hline
\end{tabular}

${ }^{a}$ Excluded ALL and brain tumour case.

Source of notifiable diseases: Centre of Health protection, Department of Health, Hong Kong Government.

The chickenpox data in year 1997 and 1998 were not available because chickenpox was not included as notifiable infectious disease till year 1999.

a

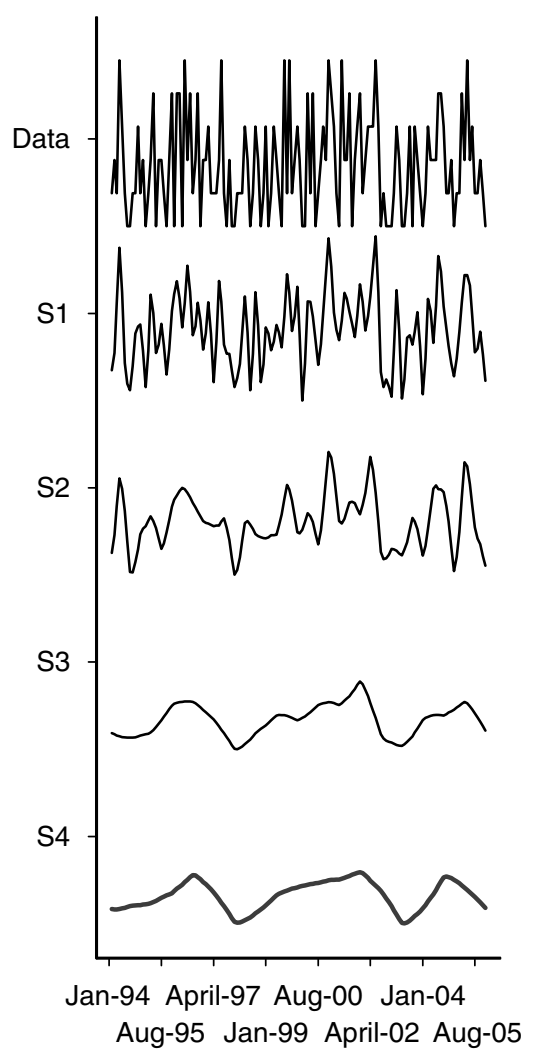

b Zooming Feature of MRA for ALL

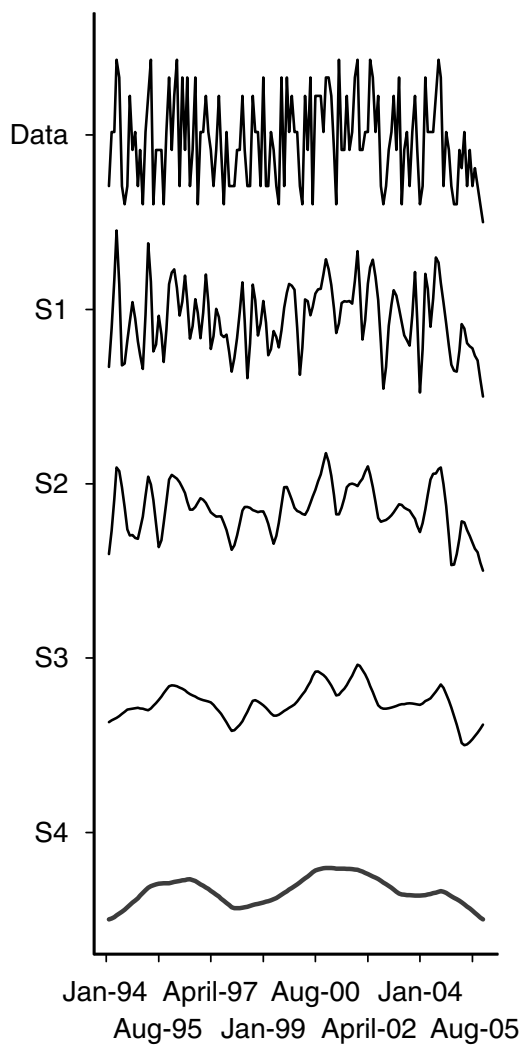

c Zooming Feature of MRA for non-ALL

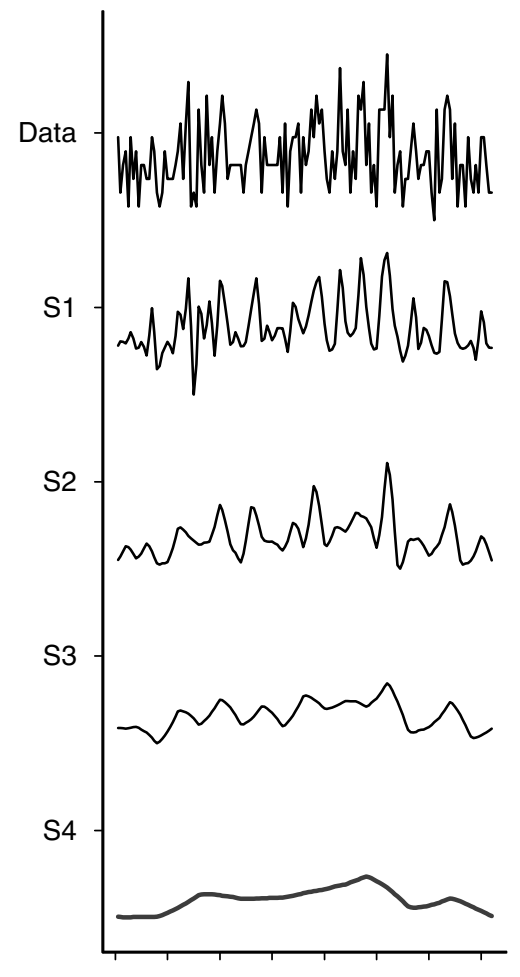

Jan-94 April-97 Aug-00 Jan-04 Aug-95 Jan-99 April-02 Aug-05

Figure $1 \quad(\mathbf{a}-\mathbf{c})$ Trend of ALL and other cancers by reconstructed multi-resolution approximations (MRA) with zooming feature of the three signals. 


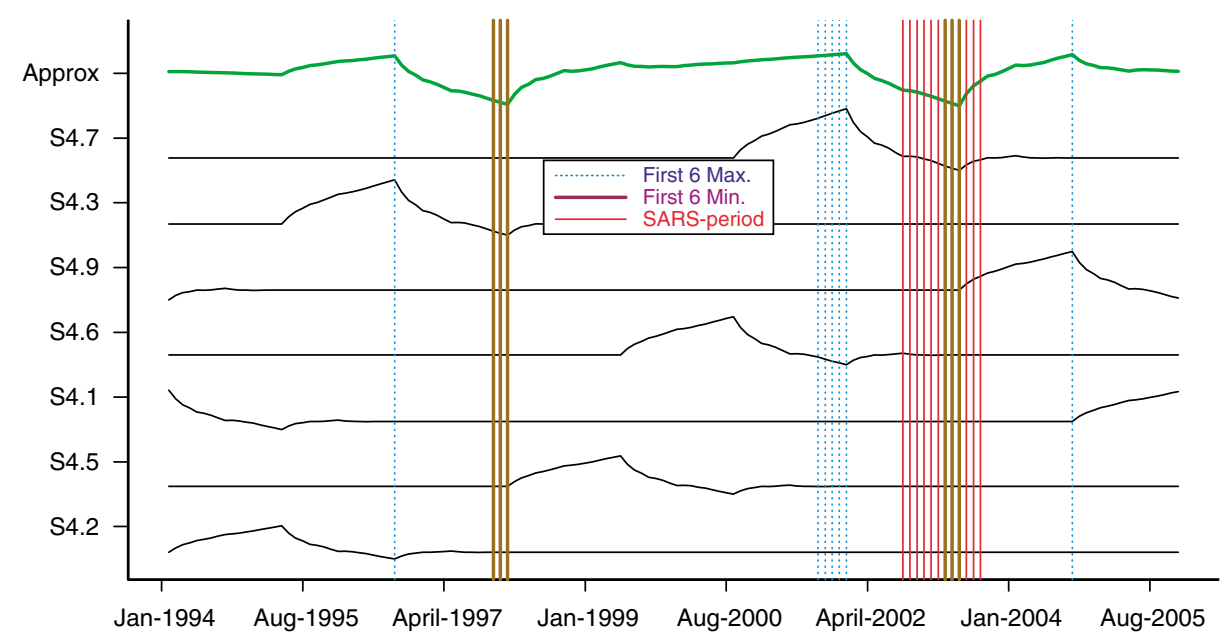

Figure 2 The decomposition of SR into seven wavelet function.

Hong Kong government from 1997 to 2005 was also studied. ${ }^{9}$ Three common childhood communicable diseases were used as reference for community-acquired infection: chickenpox, measles and scarlet fever (Table 1). There was a marked decrease of these three common infections in 2003, which then returned back to the usual trend in subsequent years. The above analysis also showed a periodic change of standard-risk ALL in 1997. Interestingly there was also an outbreak of measles in 1997 in Hong Kong together with a small cluster of avian influenza.

The epidemiological studies and laboratory studies so far supported the multiple hit theory for development of childhood ALL. An insult during pregnancy may lead to genetic predisposition that is present at birth, such as the presence of TEL/ AML1 fusion products in the cord blood. ${ }^{10} \mathrm{~A}$ second or even multiple environmental challenges including infection are required for the final step of leukaemogenesis. Greeves suggested the immunological isolation in the first year of life leading to inadequate priming of the naïve immune system, and thus making the children susceptible to infections in later years. ${ }^{11}$ The exposure of some forms of common infection may precipitate a highly dysregulated immune response and allow the proliferation of bone marrow cells and facilitate mutation. This hypothesis is supported by the protective effect of development of ALL in children with increased social activity during the first year of life such as day-care centre exposure in infancy. ${ }^{12}$ A local study in Hong Kong also demonstrated the development of roseola infection or fever with rash in infancy associated with decreased risk of developing childhood ALL. ${ }^{4}$ Another hypothesis put up by Kinlan is related to population mixing. During the influx of migrants to a relatively isolated community, there may be 'mini-epidemics' of one or more infections, to which leukaemia may be a rare response in the genetically predisposed children. ${ }^{13}$ Infection-induced leukaemia, however, has never been proved, although there are epidemiological studies supporting the hypothesis. Seasonal variability in the incidence of ALL is one of the indirect evidences. In a study conducted in Hong Kong, 'tonsillitis' 3-12 months before the diagnosis of ALL was more commonly observed in ALL cases. ${ }^{4}$ The role of infection in the pathogenesis of ALL is controversial; some studies supported while others showed protective effect or no effect. SARS is caused by coronavirus and may have haematological changes. The coronavirus, however, does not affect bone marrow cell proliferation and has not been reported to be associated with leukaemia. ${ }^{14}$ The SARS outbreak in Hong Kong in 2003, however, had a significant impact on the infection pattern of the community. The various measures combating the spread of SARS had effectively stopped the spread of SARS infection and also other types of infection. Students were isolated at home for about 2 months, and then the strict hygienic measures at school effectively reduced the spread of airborne and droplet-transmitted infections. As shown by the government reports of communicable infection from 1997 to 2005, there was a sharp decrease in many types of communicable infections. ${ }^{9}$ Chickenpox infection is a marker of airborne infection related to close contacts and mixing of children. Other infections such as scarlet fever and measles also showed marked decrease in number in 2003. The pattern of these common infections in 2003 reflected that the community was actually getting more 'clean'. We could not get accurate data on the incidence of other common childhood infections during that period as there was no such recording system. However, the notifiable diseases can serve as a marker for common communicable diseases in the community. The decrease of common communicable diseases will reflect reduced exposure of common infection in children. With the reduced exposure to infection, the genetically at risk children may be at lower risk to become targets of the second hit. The decline in the incidence of $A L L$, in particular the standard-risk ALL, in 2003 is probably the result of social isolation during SARS and thus less exposure to triggering environmental factors. We cannot exclude the possibility of non-infectious environmental factors triggering the onset of ALL. However, there were no other major physical changes in the society during the SARS period. We believe reduced exposure to infections as the most likely explanation for the change. Certainly, we cannot identify which infection is the culprit but it is probably related to one or more than one of the common childhood infections. The analysis also showed another period of low incidence of standard-risk ALL in 1997. There was an epidemic of measles in the same year. The community as a whole was more alert to the infectious disease epidemic, and parents took more precautionary measures against infection. The avian influenza cluster in the same year probably also raised the alertness of infection preventive measures in the community.

The hypothesis of naïve immune system during infancy, associated with higher incidence of ALL in later life, will be an interesting subject in the SARS setting. Babies born in 2003 had 
a much lower chance of social contacts during the SARS and post-SARS period. Will this cohort of children having a higher chance of developing ALL in later life need longer observation? Children who are at risk of developing ALL might have escaped the second hit in 2003, but it is not certain whether the risk will just be delayed for another 1-2 years. If this is the case, then we should observe a higher incidence of ALL in the subsequent few years. Since the peak age of ALL is 2-6 years, any trend of increased incidence may be detected in the coming few years. In the 2 years post-SARS period, we did not see any increase in the incidence of ALL. If children are only at risk of developing ALL secondary to infection at certain critical age such as 2-6 years, then hygienic measures to prevent common childhood infection may be one way to prevent childhood ALL.

The reduced incidence of ALL was only confined to the standard-risk ALL. The older children with ALL showed a similar incidence pattern throughout the study period. The leukaemogenesis of older children with ALL is probably different from that of younger children. Similarly, other childhood cancers also did not show significant change in the incidence pattern in the SARS period. The association of infection or environmental factors with non-ALL childhood cancers was not found in previous studies. One drawback of the study is the relatively small number of children population in Hong Kong. There are only 1.1 million children below the age of 15 years, and the annual number of new childhood ALL cases is only 30-40. It may not be easy to detect a difference in incidence with such a small number. A larger epidemiology study involving other cities severely affected by SARS may provide more information. One interesting observation in this study is the onset of ALL in relation to social isolation and probably infection. The decline in ALL cases started quite early after the institution of infection control measures. This may imply that the onset of ALL after an infectious trigger may be in terms of weeks only. So far there are no data that can follow the exposure of potential triggering factor to the clinical presentation on ALL. This study may provide indirect evidence of a rather short incubation period of ALL. In conclusion, we observed a reduced incidence of standard-risk ALL in children during the SARS period. The reduced incidence may be related to decreased exposure to common infection. This study provides further support on the hypothesis of infectious origin of childhood ALL.

\section{Acknowledgements}

We thank all members of HKPHOSG for contributing the data, and Hong Kong Children's Cancer Foundation.

CK Li ${ }^{1}, \mathrm{~B} \mathrm{Zee}^{2}$, J Lee ${ }^{3}, \mathrm{KW} \mathrm{Chik}^{1}, \mathrm{SY} \mathrm{Ha}^{4}$ and $\mathrm{V} \mathrm{Lee}{ }^{1}$, on behalf of the Hong Kong Paediatric Haematology \& Oncology Study Group

${ }^{1}$ Department of Paediatrics, Prince of Wales Hospital,
The Chinese University of Hong Kong, Hong Kong, China; ${ }^{2}$ Centre for Clinical Trials, School of Public Health, Prince of Wales Hospital, The Chinese University of Hong Kong, Hong Kong, China;

${ }^{3}$ Centre for Clinical Trials and Department of Clinical Oncology, Prince of Wales Hospital, The Chinese University of Hong Kong, Hong Kong, China and

${ }^{4}$ Department of Paediatrics and Adolescent Medicine, Queen Mary Hospital, The University of Hong Kong, Hong Kong,

China

E-mail: ckli@cuhk.edu.hk

\section{References}

1 Kinlen LJ. High-contact paternal occupations, infection and childhood leukaemia: five studies of unusual population-mixing of adults. Br J Cancer 1997; 76: 1539-1545.

2 Kinlen L, Doll R. Population mixing and childhood leukaemia: Fallon and other US clusters. Br J Cancer 2004; 91: 1-3.

3 McNallay RJQ, Eden TOB. An infectious aetiology for childhood acute leukaemia: a review of evidence. Br J Haematol 2004; 127: 243-263.

4 Chan LC, Lam TH, Li CK, Lau YL, Li CK, Yuen HL et al. Is the timing of exposure to infection a major determinant of acute lymphoblastic leukaemia in Hong Kong? Paediatr Perinat Epidemiol 2002; 16: 154-165.

5 Alexander FE, Chan LC, Lam TH, Yuen P, Leung NK, Ha SY et al. Clustering of childhood leukaemia in Hong Kong: association with the childhood peak and common acute lymphoblastic leukaemia and with population mixing. Br J Cancer 1997; 75: 457-463.

6 Lee N, Hui D, Wu A, Chan P, Cameron P, Joynt GM et al. A major outbreak of severe acute respiratory syndrome in Hong Kong. N Engl J Med 2003; 348: 1986-1994.

7 Li CK, Mang OWK, Foo W. Epidemiology of paediatric cancer in Hong Kong, 1982 to 1991. Hong Kong cancer registry. Hong Kong Med J 1999; 5: 128-134.

8 Smith M, Arthur D, Calmitta B, Caroll AJ, Crist W, Gaynon P et al. Uniform approach to risk classification and treatment assignment for children with acute lymphoblastic leukemia. J Clin Ocol 1996; 14: $18-24$.

9 Centre for Health Protection, Department of Health, The Government of Hong Kong SAR. www.chp.gov.hk/notifiable1518d.html.

10 Wiemels JL, Cazzaniga G, Daniotti M, Eden OB, Addison GM, Masera G et al. Prenatal origin of acute lymphoblastic leukaemia in children. Lancet 1999; 354: 1499-1503.

11 Greaves MF. Speculations on the cause of childhood acute lymphoblastic leukemia. Leukemia 1988; 2: 120-125.

12 Gilham C, Peto J, Simpson J, Romen E, Eden TOB, Greaves MF et al. Day care in infancy and risk of childhood acute lymphoblastic leukaemia: findings from UK case-control study. BMJ 2005; 330: 1279-1280.

13 Kinlen LJ. Epidemiological evidence for an infective basis in childhood leukemia. B J Cancer 1995; 17: 1-5.

14 Ding Y, He L, Zhang Q, Huang Z, Che X, Hou J et al. Organ distribution of severe acute respiratory syndrome (SARS) associated coronavirus (SARS-CoV) in SARS patients: implications for pathogenesis and virus transmission pathways. J Pathol 2004; 203: 622-630.

Supplementary Information accompanies the paper on the Leukemia website (http://www.nature.com/leu) 